\title{
CLOSED 3-MANIFOLDS WITH NO PERIODIC MAPS
}

\author{
BY \\ FRANK RAYMOND AND JEFFREY L. TOLLEFSON $\left({ }^{\mathbf{1}}\right)$
}

\begin{abstract}
Examples of closed, orientable, aspherical 3-manifolds are constructed on which every action of a finite group is trivial.
\end{abstract}

1. Introduction. In this paper we give examples of closed, orientable, aspherical 3-manifolds on which every action of a finite group is trivial. These 3-manifolds can be fibered over $S^{1}$ with fiber a closed orientable surface of genus greater than or equal to 3 . For such examples, three is the smallest possible genus of the fiber since every 3-manifold fibered over $S^{1}$ with a fiber of genus $\leqslant 2$ admits nontrivial involutions [1].

P. E. Conner and F. Raymond [4] first constructed examples of manifolds exhibiting this total lack of nontrivial periodic homeomorphisms. Their examples were compact nonaspherical 4-manifolds with boundary. We use some of the methods introduced in that paper which provide information as to which finite groups can act effectively on certain manifolds. The starting point is the following theorem of A. Borel. Let $G$ be a group of homeomorphisms of a closed, connected, aspherical manifold $M$. Consider the homomorphism $\psi: G \rightarrow$ Out $\left(\pi_{1}(M)\right)$ which sends $g \in G$ to the outer automorphism of $\pi_{1}(M)$ induced by the homeomorphism $g$. Then if $\pi_{1}(M)$ has trivial center, $\psi$ is a monomorphism. We obtain our results by proving that for certain 3-manifolds this group of outer automorphisms is torsion free.

Conner, Raymond, and Weinberger in [5] construct closed aspherical manifolds (of dimensions 7,11, 16, 22, 29, and 37) which admit no effective action of any nontrivial group. Also E. Bloomberg in [2] has constructed nonaspherical, closed, orientable 4-manifolds on which no finite group can operate.

The examples presented in this paper are the first 3-manifolds known to admit no periodic maps at all. This result in the PL category was first observed by the second author [10], whereas now we work in the topological category.

We wish to thank Professor Joan Birman for some helpful observations concerning surface maps.

Received by the editors February 28, 1975 and, in revised form, July 10, 1975.

AMS (MOS) subject classifications (1970). Primary 57A10, 57E25; Secondary 55C35.

Key words and phrases. Periodic maps, 3-manifolds.

$\left({ }^{1}\right)$ Both authors were supported in part by the National Science Foundation. 
2. The examples. In this section we construct a family of 3-manifolds $M_{\Phi}$ which admit no nontrivial periodic maps. The proof that these 3-manifolds have this property is sketched here with the details occupying the remaining three sections.

We use the notion of a twist map [8] in defining the surface homeomorphism $\Phi$ used for the construction of $M_{\Phi}$. Let $c$ denote a simple closed curve on the closed, orientable surface $F$. A twist $t(c)$ about $c$ is defined in the following way. Let $A$ denote a regular neighborhood of $c$ and assume that the annulus $A$ is parametrized by $(r, \theta),-1 \leqslant r \leqslant 1,0 \leqslant \theta \leqslant 2 \pi$. A twist $t(c)$ is defined by $t(c)(r, \theta)=(r, \theta+\pi(r+1))$ in $A$ and by the identity outside $A$. The sense of $t(c)$ depends only on the choice of a positive direction for $\theta$. We adopt the convention that the parametrization of $A$ is always chosen such that $t(c)$ moves points on a directed line segment which is approaching $c$ to the right. The isotopy class of $t(c)$ is invariant under isotopic deformations of the curve $c$.

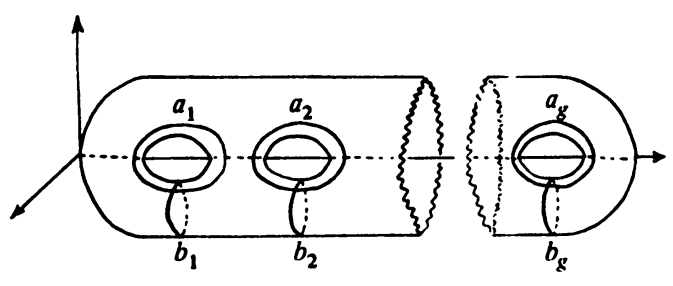

Figure 1

Now consider a closed orientable surface $F$ of genus $g(g \geqslant 3)$ embedded in $R^{3}$ as illustrated in Figure 1 and let $\left\{a_{i}, b_{i}: 1 \leqslant i \leqslant g\right\}$ denote the set of oriented simple closed curves shown. The first homology group $H_{1}(F)$ is a free abelian group with $2 g$ generators $\left\{\hat{a}_{i}, \hat{b}_{i}\right\}$ corresponding to the curves $\left\{a_{i}, b_{i}\right\}$. Let us look at the automorphisms of $H_{1}(F)$ induced by the twist maps $t\left(a_{i}\right)$ and $t\left(b_{i}\right)^{-1}$. Each generator is fixed by $t\left(a_{i}\right)_{*}$ except $\hat{b}_{i}$ which is mapped to $\hat{a}_{i} \hat{b}_{i}$ and $t\left(b_{i}\right)_{*}^{-1}$ sends $\hat{a}_{i}$ to $\hat{a}_{i} \hat{b}_{i}$ and fixes the remaining generators.

Fix a set $\left\{n_{1}, \ldots, n_{g}\right\}$ of arbitrary but distinct positive integers, each greater than 2, and define $\Phi$ to be the homeomorphism of the surface $F$ obtained by setting $\Phi=\prod_{i=1}^{g} t\left(a_{i}\right) t\left(b_{i}\right)^{-n_{i}+1}$. Now on $R^{1} \times F$ we define an action of the integers $Z$ as a group of covering transformations by $n(t, x)=\left(t-n, \Phi^{n}(x)\right)$. Our examples $M_{\Phi}$ are taken to be the resulting quotient spaces of the action and thus are closed, aspherical, orientable 3-manifolds. We write the points of $M_{\Phi}$ as $[t, x]$. There is a fibering $p: M_{\Phi} \rightarrow S^{1}$, defined by $p([t, x])=\exp (2 \pi i t)$, with fiber $F$. We may view $\pi_{1}\left(M_{\Phi}\right)$ as the semidirect product $\pi_{1}(F) \circ Z$ determined by the automorphism of $\pi_{1}(F)$ induced by $\Phi$.

We are going to prove some things about $\operatorname{Out}\left(\pi_{1}(F)\right)$, the group of outer automorphisms of $\pi_{1}(F)$, and in the process we will need some information about 
the automorphism $\Phi_{*}$ of $H_{1}(F)$ induced by the map $\Phi$. The matrix $P$ in $G L(2 g, Z)$ corresponding to this automorphism $\Phi_{*}$ with respect to the generators $\left\{\hat{a}_{i}, \hat{b}_{i}\right\}$ is

$$
P=\left(\begin{array}{ccccc}
X\left(n_{1}\right) & 0 & 0 & \ldots & 0 \\
0 & X\left(n_{2}\right) & 0 & \ldots & 0 \\
\vdots & & & & \\
0 & 0 & 0 & \ldots & X\left(n_{g}\right)
\end{array}\right)
$$

shown by blocks of $2 \times 2$ matrices, where $X(n)=\left(\begin{array}{cc}n & 1 \\ n-1 & 1\end{array}\right)$. This matrix $P$ will be considered in the next section.

Now we are ready to take up our main result.

THEOREM A. There exist no effective actions on $M_{\Phi}$ by any nontrivial finite group.

Proof. Observe that the group $\pi_{1}\left(M_{\Phi}\right)$ is centerless (see for example Theorem 4.7 of [4]). Now by Borel's theorem, if the group $G$ acts effectively on $M_{\Phi}$, then the representation $\operatorname{Homeo}\left(M_{\Phi}\right) \rightarrow \operatorname{Out}\left(\pi_{1}\left(M_{\Phi}\right)\right)$ must be a monomorphism when restricted to $G$. Our theorem will then follow after we show that $\operatorname{Out}\left(\pi_{1}\left(M_{\Phi}\right)\right)$ is a torsion free group. For this we proceed as follows.

First, since $\operatorname{det}(I-P)=\Pi\left(n_{i}-1\right) \neq 0$, the results in $\S 4$ of [4] can be applied to study Out $\left(\pi_{1}\left(M_{\Phi}\right)\right)$. Let $\Phi_{\#}$ denote the outer automorphism of $\pi_{1}(F)$ determined by the map $\Phi$ and let $\left(\Phi_{\#}\right)$ be the infinite cyclic subgroup of

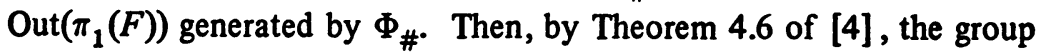
$\operatorname{Out}\left(\pi_{1}\left(M_{\Phi}\right)\right)$ is isomorphic to the quotient group $N\left(\Phi_{\#}\right) /\left(\Phi_{\#}\right)$ where $N\left(\Phi_{\#}\right)$ denotes the normalizer of $\left(\Phi_{\#}\right)$ in $\operatorname{Out}\left(\pi_{1}(F)\right)$.

We shall prove in $\S 4$ that $N\left(\Phi_{\#}\right) /\left(\Phi_{\#}\right)$ has no elements of odd order. For this we project $N\left(\Phi_{\#}\right) /\left(\Phi_{\#}\right)$ into $N(P) /(P)$, where $N(P)$ is the normalizer of the infinite cyclic group generated by $P$ in $G L(2 g, Z)$. In $\$ 3$ we show that $N(P) /(P)$ $\cong Z^{g-1} \oplus\left(Z_{2}\right)^{g}$. Then we prove that the kernel of this projection has no elements of finite order. The existence of such an element of finite order in this kernel would imply that some periodic map of $F$ acts trivially on homology, which is impossible. It also follows from this argument that any element of order two in $N\left(\Phi_{\#}\right) /\left(\Phi_{\#}\right)$ must be induced by an involution of $F$ which commutes with $\Phi$ up to homotopy. The proof is completed in $\$ 5$, where we show that no such involution of $F$ can exist.

3. The matrix $P$. In the above section we introduced the matrix $\boldsymbol{P}$ corresponding to the isomorphism $\Phi_{*}$ of $H_{1}(F)$. We let $(P)$ denote the infinite cyclic subgroup of $G L(2 g, Z)$ generated by $P$ and write the centralizer and normal- 
izer of $(P)$ in $G L(2 g, Z)$ as $C(P)$ and $N(P)$, respectively. The content of this section is practically the same as that of the early part of \$4 in [4] where matrices of the form $\left(\begin{array}{cc}n-1 & 1 \\ n & 1\end{array}\right)$ instead of $\left(\begin{array}{cc}n & 1 \\ n-1 & 1\end{array}\right)$ are used. We use the latter type in order that the 3-manifold examples we obtain be orientable.

The object of this section is to establish the next theorem. Once we have Lemma 7 below, its proof follows from the arguments used to prove the analogous result in [4] (see the discussion there on p. 39 and Theorem 6.7).

Theorem B. (i) The matrix $P$ has no roots in $G L(2 g, Z)$. (ii) $N(P)=$ $C(P)$ and they are isomorphic to $Z^{g} \oplus\left(Z_{2}\right)^{g}$, where $(P)$ corresponds to the diagonal subgroup of $Z^{g}$. (iii) $N(P) /(P)$ is isomorphic to $Z^{g-1} \oplus\left(Z_{2}\right)^{g}$.

We first establish some properties of the matrix $X(n)=\left(\begin{array}{cc}n & 1 \\ n-1 & 1\end{array}\right)$, where $n$ is assumed to be greater than 2 throughout.

LEMMA 1. There does not exist a matrix $B \in G L(2, Z)$ for which $X(n) B$ $=B X(n)^{-1}$.

Proof. Consider the products

$$
\left(\begin{array}{cc}
n & 1 \\
n-1 & 1
\end{array}\right)\left(\begin{array}{ll}
a & b \\
c & d
\end{array}\right)=\left(\begin{array}{cc}
a n+c & b n+d \\
a(n-1)+c & b(n-1)+c
\end{array}\right)
$$

and

$$
\left(\begin{array}{ll}
a & b \\
c & d
\end{array}\right)\left(\begin{array}{cc}
1 & -1 \\
1-n & n
\end{array}\right)=\left(\begin{array}{ll}
a+b(1-n) & -a+b n \\
c+d(1-n) & -c+d n
\end{array}\right)
$$

From $b n+d=-a+b n$ we see that $a=-d$, and $b(n-1)+c=-c+d n$ implies that $2 c=d n+b(1-n)$. Hence $2 c=-a n+b(1-n)$. Now from $a n+c$ $=a+b(1-n)$ we have $c=a-a n+b(1-n)$. Eliminating $c$ from these two equations gives us $a(2-n)+b(1-n)=0$. Therefore

$$
B=\left(\begin{array}{cc}
b(n-1) /(2-n) & b \\
b /(2-n) & -b(n-1) /(2-n)
\end{array}\right) .
$$

Then

$$
|\operatorname{det} B|=b^{2}\left(\frac{n-1}{n-2}\right)\left(\frac{2 n-3}{n-2}\right)>b^{2} .
$$

Since $\mid$ det $B \mid=1$ implies that $b=0$, we have a contradiction.

LEMMA 2. The centralizer of $\left(\begin{array}{cc}n & 1 \\ n-1 & 1\end{array}\right)$ in $G L(2, Z)$ is the subgroup of matrices of the form

$$
\left(\begin{array}{cc}
d+b(n-1) & b \\
b(n-1) & d
\end{array}\right)
$$

where $d^{2}+b d(n-1)=b^{2}(n-1) \pm 1$. 
Proof. Follows from direct calculation.

LEMMA 3 [4]. If $B=\left(\begin{array}{ll}a & b \\ c & d\end{array}\right) \in G L(2, Z)$ is a matrix for which $a d>0, b c$ $>0$ then exactly one of the four matrices $B,-B, B^{-1},-B^{-1}$ has all strictly positive entries.

Proof. Easily verified by checking the few possibilities.

LEMMA 4. If $k>1$ then there is no matrix $B \in G L(2, Z)$ for which $B^{k}=$ $\left(\begin{array}{cc}n-1 & 1 \\ n-1\end{array}\right)$.

Proof. Suppose that a matrix $B$ does exist for which $B^{k}=\left(\begin{array}{cc}n & 1 \\ n-1 & 1\end{array}\right)$. Then $B$ commutes with $X(n)$ and we see from Lemma 2 that $d^{2}+b d(n-1)=$ $b^{2}(n-1) \pm 1$. If $b=0$, then $B^{2}=I$. Thus, we may assume that $b \neq 0$. Since $n>2$ we notice that $d^{2}+b d(n-1)>0$ and Lemma 3 applies. Let $C$ denote the one matrix $B,-B, B^{-1}$, or $-B^{-1}$ with all entries strictly positive. Then we have

$$
C^{k}= \pm\left(\begin{array}{cc}
n & 1 \\
n-1 & 1
\end{array}\right)^{ \pm 1}
$$

which is impossible for $k>1$.

LEMMA 5. If $B$ is in the centralizer of $\left(\begin{array}{cc}n & 1 \\ n-1 & 1\end{array}\right)$ in $G L(2, Z)$ and $B^{k}=I$ $(k \geqslant 1)$, then $B= \pm I$.

Proof. Very similar to the proof of Lemma 4.

LEMMA 6. If $m \neq n$, then $Y=\left(\begin{array}{ll}0 & 0 \\ 0 & 0\end{array}\right)$ is the only solution for either of the equations $Y X(n)=X(m) Y$ or $Y X(n)=X(m)^{-1} Y$.

Proof. Let $Y=\left(\begin{array}{ll}a & b \\ c & d\end{array}\right)$. By computing both sides of the equation $Y N(n)$ $=X(m) Y$ we obtain

$$
\left(\begin{array}{ll}
a n+b(n-1) & a+b \\
c n+d(n-1) & c+d
\end{array}\right)=\left(\begin{array}{cc}
a m+b & b m+d \\
a(m-1)+b & b(m-1)+d
\end{array}\right) .
$$

From the equation $c+d=b(m-1)+d$ we get $c=(m-1) b$, which together with $a m+b=c n+d(n-1)$ implies that $a=-b$. Now from $a+b=b m+d$ we see that $d=m a$. Finally, from $c n+d(n-1)=a(m-1)+b$ we obtain $(n-1) a=(m-1) a$, which implies that $a=0$. Therefore $Y=\left(\begin{array}{ll}0 & 0 \\ 0 & 0\end{array}\right)$.

Let us turn to the product

$$
\left(\begin{array}{cc}
1 & -1 \\
1-m & m
\end{array}\right)\left(\begin{array}{ll}
a & b \\
c & d
\end{array}\right)=\left(\begin{array}{cc}
a-c & b-d \\
a(1-m)+c m & b(1-n)+d m
\end{array}\right)
$$

and consider the equation $Y X(n)=X(m)^{-1} Y$. From $a+b=b-d$ we get $a=-d$, and from $a-c=a n+b(n-1)$ we obtain $c=(1-n)(a+b)$. On the 
other hand, $c+d=b(1-m)+d m$ implies $c=(a+b)(1-m)$. Thus $(a+b)(1-m)=(1-n)(a+b)$. Since $m \neq n$, we see that $a=-b$ and $c=0$. But from $c n+d(n-1)=a(1-m)+c m$ we obtain $a=c$. Therefore $Y=$ $\left(\begin{array}{ll}0 & 0 \\ 0 & 0\end{array}\right)$.

Let $A=\left(a_{i j}\right)$ denote an arbitrary $(2 g \times 2 g)$ matrix in $G L(2, Z)$, where the $a_{i j}$ are $2 \times 2$ matrices and $1 \leqslant i, j \leqslant g$.

LEMMA 7. (i) If $A$ has finite order and $A P=P A$ then $a_{i j}=\left(\begin{array}{ll}0 & 0 \\ 0 & 0\end{array}\right)$ for $i \neq$ $j$ and $a_{i i}= \pm\left(\begin{array}{ll}1 & 0 \\ 0 & 1\end{array}\right)$ for $1 \leqslant i, j \leqslant g$. (ii) If $A^{k}=P$ for a positive integer $k$, then $k=1$. (iii) $A P \neq P^{-1} A$.

Proof. Suppose $A^{k}=I(k \geqslant 1)$ and $A P=P A$. We obtain from the latter equation the equations (i) $a_{i j} X\left(n_{j}\right)=X\left(n_{i}\right) a_{i j}$ (for $i \neq j$ ) and (ii) $a_{i i} X\left(n_{i}\right)$ $=X\left(n_{i}\right) a_{i i}$. We see from Lemma 6 that equation (i) implies $a_{i j}=\left(\begin{array}{ll}0 & 0 \\ 0 & 0\end{array}\right)$ for $i \neq$ $j$. Thus for each $i$ we have $a_{i i} \in G L(2, Z)$ and $a_{i i}^{k}=I$. It follows from Lemma 5 that $a_{i i}= \pm I$.

Suppose that $A^{k}=P$ for some positive integer $k$. As before, we conclude from the fact that $A$ commutes with $P$ that $a_{i j}=\left(\begin{array}{ll}0 & 0 \\ 0 & 0\end{array}\right)$ for $i \neq j$. Thus $a_{i i} \in$ $G L(2, Z)$ and $a_{i i}^{k}=X\left(n_{i}\right)$. Then Lemma 4 gives us $k=1$.

Observe that $A P=P^{-1} A$ implies that $a_{i j} X\left(n_{j}\right)=X\left(n_{i}\right)^{-1} a_{i j}$. Thus if $i \neq j$ we must have $a_{i j}=\left(\begin{array}{ll}0 & 0 \\ 0 & 0\end{array}\right)$. Hence $a_{i i} \in G L(2, Z)$ and it follows from Lemma 1 that $a_{i i} X\left(n_{i}\right) \neq X\left(n_{i}\right)^{-1} a_{i i}$. This completes the proof.

4. Torsion in $N\left(\Phi_{\#}\right) /\left(\Phi_{\#}\right)$. Now we show that if the group $N\left(\Phi_{\#}\right) /\left(\Phi_{\#}\right)$ has an element of finite order, then this element is induced by an involution of $F$ that commutes with $\Phi$ up to homotopy. Some information about this group can be obtained by considering the image of $N\left(\Phi_{\#}\right)$ under the homomorphism $\operatorname{Out}\left(\pi_{1}(F)\right) \rightarrow \operatorname{Aut}\left(H_{1}(F)\right)$ and using the results of the previous section. The first lemma is surely well known. However we include a proof since we are unaware of its appearance elsewhere in the literature.

LEMMA 8. Let $F$ be a closed orientable surface of genus $g>1$. If $G$ is a finite group of homeomorphisms of $F$ then under the composition of homomorphisms

$$
\operatorname{Homeo}(F) \rightarrow \operatorname{Out}\left(\pi_{1}(F)\right) \rightarrow \operatorname{Aut}\left(H_{1}(F)\right)
$$

the restriction to $G$ is a monomorphism.

Proof. One need only observe that if $h$ is a periodic map of $F$ of prime period $p$ then $h_{*} \in \operatorname{Aut}\left(H_{1}(F)\right)$ also has period $p$. This is equivalent to showing that $h_{*} \neq 1$.

Case 1. Suppose that $h$ is an orientation-reversing involution of $F$. Choose 
two oriented curves $a, b$ with intersection number one which represent two of the generators $\hat{a}, \hat{b}$ of $H_{1}(F)$. Then $\hat{a} \cdot \hat{b}=-h_{*}(\hat{a}) \cdot h_{*}(\hat{b})$. If both $h_{*}(\hat{a})=\hat{a}$ and $h_{*}(\hat{b})=\hat{b}$ then we have a contradiction. Therefore $h_{*} \neq 1$.

Case 2. Suppose that $h$ is an orientation-preserving map of prime period $p$. Let $f: F \rightarrow F^{*}$ denote the projection onto the orbit space $F^{*}$ of the cyclic action on $F$ generated by $h$. Recall that $H^{n}\left(F^{*} ; Q\right)$ is isomorphic to $\left[H^{n}(F ; Q)\right]^{n^{*}}$, the subgroup of $H^{n}(F ; Q)$ consisting of all elements fixed by $h^{*}$ (see Corollary 2.3 , p. 38 of [3]). Then by duality we obtain $H_{1}\left(F^{*} ; Q\right) \cong$ $\left[H_{1}(F ; Q)\right]^{\text {h* }}$.

If $\operatorname{Fix}(h)=\varnothing$ then $\chi(F)=p \chi\left(F^{*}\right)$. Thus $(2-2 g)=p\left(2-2 g^{*}\right)$, where $g^{*}$ denotes the genus of $F^{*}$. It follows that $g^{*}<g$ and hence $\operatorname{dim} H_{1}\left(F^{*} ; Q\right)<$ $\operatorname{dim} H_{1}(F ; Q)$. Hence we cannot have $h_{*}=1$.

Now assume $\operatorname{Fix}(h) \neq \varnothing$. By Smith theory we have $\chi(F)=(1-p) x(\operatorname{Fix}(h))$ $+p \chi\left(F^{*}\right)$. This gives us the equation $(2-2 g)=(1-p) t+p\left(2-2 g^{*}\right)$, where $t=\chi\left(F^{*}\right)$. Again it follows that $g^{*}<g$ and therefore $h_{*} \neq 1$.

Before we proceed with the next lemma we wish to point out that $N\left(\Phi_{\#}\right)$ $=C\left(\Phi_{\#}\right)$ in $\operatorname{Out}\left(\pi_{1}(F)\right)$. Since $\left(\Phi_{\#}\right)$ is an infinite cyclic subgroup, this is equivalent to saying there does not exist any $\Psi \in \operatorname{Out}\left(\pi_{1}(F)\right)$ such that $\Psi \Phi_{\#} \Psi^{-1}=$ $\Phi_{\#}^{-1}$. But this follows by projecting $\operatorname{Out}\left(\pi_{1}(F)\right)$ into $\operatorname{Auto}\left(H_{1}(F)\right)$ and applying Theorem B.

LEMma 9. The group $N\left(\Phi_{\#}\right) /\left(\Phi_{\#}\right)$ has no element of odd prime order and any element of order two is induced by an involution of $F$ which commutes with $\Phi$ up to homotopy.

Proof. Suppose that there exists an element of $N\left(\Phi_{\#}\right) /\left(\Phi_{\#}\right)$ of prime order $p$. Then there exists $\gamma \in C\left(\Phi_{\#}\right)=N\left(\Phi_{\#}\right)$ such that $\gamma^{p}=\Phi_{\#}^{k}$ for some $k$ and $\gamma \notin\left(\Phi_{\#}\right)$. We consider three cases.

Case 1. $k>0$ and $(p, k)=1$. There exist integers $a, b$ such that $a p+b k$ $=1$. From the equation $\gamma_{*}^{p}=\Phi_{*}^{k}$ we obtain $\left(\Phi_{*}^{a} \gamma_{*}^{b}\right)^{p}=\Phi_{*}$, which is a contradiction since $\Phi_{*}$ has no roots.

Case 2. $k=0$. In this case $\gamma^{p}=1$. By a theorem of Nielsen [9] there is a homeomorphism $\alpha$ of $F$ such that $\alpha$ induces $\gamma$ and $\alpha^{p}=1$. By Lemma 8 we see that $\gamma_{*}$ is not the identity in $\operatorname{Aut}\left(H_{1}(F)\right.$ ). But then $p=2$ (by Theorem B) and $\gamma \Phi_{\#} \gamma^{-1}=\Phi_{\#}$. Therefore $\alpha$ is an involution and $\alpha \Phi \alpha^{-1}$ is homotopic to $\Phi$.

Case 3. $k=p s>0$. From the equation $\gamma^{p}=\left(\Phi_{\#}^{s}\right)^{p}$ we obtain $\left(\gamma \Phi_{\#}^{-s}\right)^{p}=$ 1. By Nielsen's theorem again we obtain a homeomorphism $\alpha$ of $F$ such that $\alpha^{p}=1$ and $\alpha$ induces $\gamma \Phi_{\#}^{-s}$. As before, $\alpha_{*} \neq 1$ and $p=2$. It follows that $\alpha$ is an involution of $F$ and $\alpha \Phi \alpha^{-1}$ is homotopic to $\Phi$. 
5. The map $\Phi$. In this section we deal strictly with maps of 2-manifolds. Recall from $\$ 2$ the map $\Phi$ of the closed orientable surface $F$ of genus $g$ is given by $\Pi_{i=1}^{g} t\left(a_{i}\right)\left(t\left(b_{i}\right)\right)^{-n_{i}+1}$, where $\left(n_{1}, \ldots, n_{g}\right)$ is a set of $g$ distinct integers each greater than 2 and $g \geqslant 3$.

THEOREM C. The map $\Phi$ is not homotopic to $\alpha \Phi \alpha^{-1}$ for any nontrivial involution $\alpha$ of $F$.

The result we have been after now follows immediately from Lemma 9.

COROLlary. The group $\operatorname{Out}\left(\pi_{1}\left(M_{\Phi}\right)\right) \cong N\left(\Phi_{\#}\right) /\left(\Phi_{\#}\right)$ is torsion free.

First we establish some lemmas leading up to the proof of the theorem. Let us set some notation. Let $\Gamma$ denote the graph $\bigcup_{i=1}^{g}\left(a_{i} \cup b_{i}\right)$, which has $g$ figure-eight components. Let $A_{i}=a_{i} \times I$ and $B_{i}=b_{i} \times I$ denote small regular neighborhoods of the simple closed curves $a_{i}=a_{i} \times 1 / 2$ and $b_{i}=b_{i} \times 1 / 2$, respectively, such that $A_{i} \cap B_{i}$ is a disk and $\left(A_{i} \cup B_{i}\right) \cap\left(A_{j} \cup B_{j}\right)=\varnothing$ whenever $i \neq j$. Define $A=\bigcup A_{i}$ and $B=\bigcup B_{i}$. We may assume that the twist maps used in the definition of $\Phi$ are each constant outside $A \cup B$. Let $\alpha$ denote an arbitrary, but fixed, involution of $F$. We say the graph $\Gamma$ is in $\alpha$-general position if (i) $\Gamma$ meets $\operatorname{Fix}(\alpha)$ transversally, (ii) $\Gamma-\operatorname{Fix}(\alpha)$ is in general position with respect to $\alpha(F)-F i x(\alpha)$, and (iii) $\Gamma \cap \alpha(\Gamma)$ contains no vertices of either graph.

LEMMA 10. Let $c$ be a simple closed curve in $F$ such that $c$ meets $\Gamma$ transversally, $c$ separates $F$, and $\Phi(c)$ is homotopic to $c$ in $F$. If $c \cap \Gamma \neq \varnothing$ then corresponding to some $a_{i}$ or $b_{i}$, which we denote by $x$, there exists a disk $E$ in $F$ such that $E \cap(c \cup x)=\partial E$ and $\partial E=s \cup t$, where $s$ is an arc in $c$ and $t$ is an arc in $x$ with $\partial t=\partial s=s \cap t$. Moreover, $s \cap \Gamma-s \cap x$ is either empty or one point.

Proof. Let $U=c \times I$ be a small regular neighborhood of $c=c \times 1 / 2$ such that $U \cap(A \cup B)$ is a regular neighborhood of $c \cap \Gamma$ and $U \cap A \cap B=\varnothing$. For each component $V$ of $U \cap(A \cup B)$ we may assume that either the arc $V \cap$ $(c \times j)$ meets the arc $V \cap\left(a_{i} \times k \cup b_{i} \times k\right)$ in a single point transversally or the latter set is vacuous (for $1 \leqslant i \leqslant g$ and $0 \leqslant j, k \leqslant 1$ ). We let $d$ denote the simple closed curve $c \times 3 / 4$ and define $c^{\prime}=\Phi(d)$. Notice that we can decompose $c^{\prime}$ into the union of $d-(A \cup B)$ and $\bigcup \Phi\left(k_{\lambda}^{\prime}\right)$, where $k_{\lambda}^{\prime}$ ranges over the components of $d \cap(A \cup B)$. We let $k_{\lambda}$ denote the components of $c \cap(A \cup B)$ corresponding to $k_{\lambda}^{\prime}$. We illustrate in Figures $3 \mathrm{a}$ and 5 the two situations arising when $c$ cuts across both $a_{i}$ and $b_{i}$.

In Figure $3 \mathrm{a}$ notice that there is a disk $D$ whose boundary is contained in $c \cup c^{\prime}$ but whose existence is independent of the fact that $c$ is homotopic to $c^{\prime}$. Our plan is to push $c^{\prime}$ across such nonessential disks by an isotopy in order to obtain a more useful simple closed curve $c^{\prime \prime}$. 
Consider a pair of arcs $s$ and $t$ in $A \cup B \cup U$. We shall refer to them as being neighbors if $s$ and $t$ are unions of corresponding pairs of parallel arc in the components of $(A \cup B \cup U)-((A \cap B) \cup(A \cap U) \cup(B \cap U)), A \cap B$, and $(A \cup B) \cap U$. For example, $k_{\lambda}$ and $k_{\lambda}^{\prime}$ are trivially neighbors. We break the construction of the required isotopy into four steps. To ease notation, we shall continue to use $c^{\prime}$ to denote the image of $c^{\prime}$ after deforming it by an isotopy.

Step 1. Suppose there exists a disk $D \subset U$ such that $D \cap\left(c \cup c^{\prime}\right)=\partial D=$ $s \cup t$, where $\partial s=\partial t=s \cap t$ and $s$, an arc in $c$, is a neighbor of $t$, an arc in $c^{\prime}$ (see Figure 2a). By an isotopy constant outside a small regular neighborhood $V$ of $D(V \subset U)$, move $t$ across $D$ to a neighbor of $s$ on the opposite side of $c$ (see Figure 2b). After this isotopy we have $c \cap c^{\prime} \cap V=\varnothing$. We perform this operation wherever possible before proceeding.

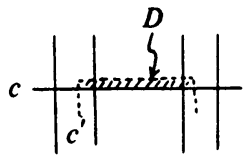

(a)

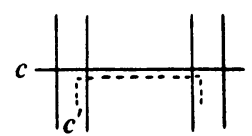

(b)

FIGURE 2

Step 2. Suppose that there exists a disk $D$ such that $D \cap(\Gamma \cup c)=\partial D=$ $s \cup t$, where $s$ is an arc in $c, t$ is an arc in $\Gamma$ containing some $a_{i} \cap b_{i}$, and $\partial s=$ $\partial t=s \cap t$ (see Figure 3a). We further suppose that there exist subarcs $\{q\}$ of $c^{\prime}$ such that each arc $q$ is a neighbor of $t$ and $\partial q=q \cap c$. Let $Q$ denote the union of these arcs $\{q\}$. By an isotopy, constant outside a regular neighborhood $V$ of $D$ containing $Q$, we move $Q$ across $D$ to a family of arcs on the opposite side of $c$ which-are neighbors of $S$ (see Figure $3 \mathrm{~b}$ ). At this point we repeat Step 1 if it is applicable. We exhaust all possible applications of Step 2 before proceeding further.

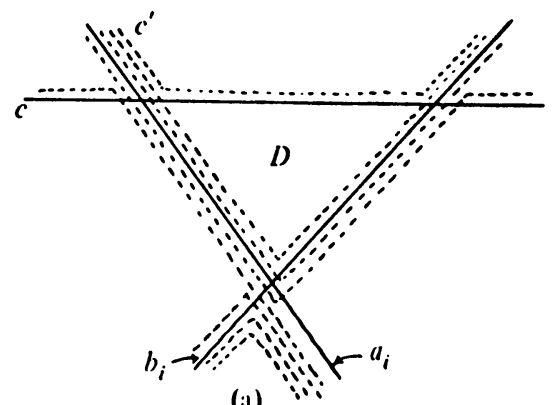

(a)

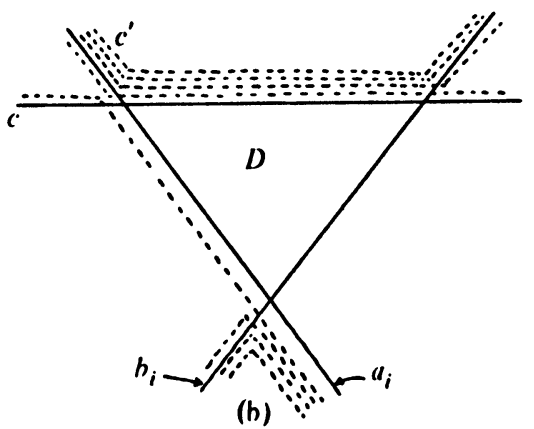

(b)

Figure 3

Step 3. Suppose there exists a disk $D$ such that (i) $\partial D=s \cup t=$ $D \cap\left(c \cup c^{\prime}\right)$, where $s$ is a subarc of $c$, the arc $t$ is contained in $c^{\prime}$, and $s \cap t=$ $\partial s=\partial t$, (ii) $s$ is not contained in $A \cup B$, and (iii) $t$ is not a neighbor of any 
subarc of $s$ (see Figure 4). Let $V$ denote a small regular neighborhood of $D$ such that $V \cap\left(c \cup c^{\prime}\right)$ is a regular neighborhood of $s \cup t$ in $c \cup c^{\prime}$. By an isotopy constant outside $V$, we move $t$ across $D$ to an arc that is a neighbor of $s$ on the opposite side of $c$. We repeat this construction as often as possible. It is likely that we shall be required to push across a given disk several times before we are finished. Repeat Step 1 if it is applicable before going on.

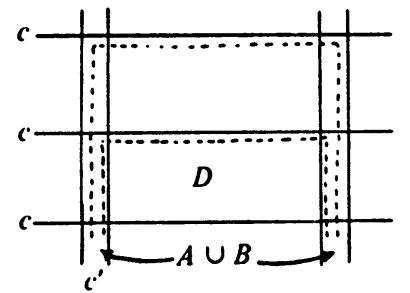

(a)

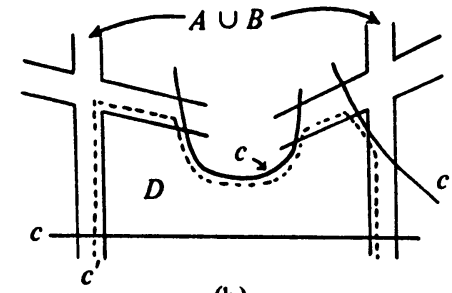

(b)

\section{Figure 4}

Step 4. Suppose there exists a disk $D$ such that $D \cap(\Gamma \cup c)=\partial D=s \cup$ $t$, where $s$ is a subarc of $c$, the arc $t$ is a subarc of $\Gamma$ containing one of the points $a_{i} \cap b_{i}$, and no subarc of $c^{\prime}$ is a neighbor of $t$ (see Figure 5). Let $V$ denote a small regular neighborhood of $s$ in $U$. Observe there exists a subarc $p$ of $c^{\prime}$ such that $p \subset A_{i} \cup B_{i} \cup V$, the arc $p$ has one endpoint in $c$ and the other in $\partial A_{i}$, and $p$ can be viewed as the union of arcs $p_{1} \cup p_{2} \cup p_{3}$, where $p_{1}$ is a neighbor of $\partial D-\{$ a point $\}, p_{2}$ and $p_{3}$ are neighbors of each other contained in $A_{i}$. We push the $\operatorname{arc} p$ across $D$ and back along $p_{2} \cup p_{3}$ to an $\operatorname{arc} q$ in the disk $U \cap A_{i}$ such that $\partial q=\partial p$ and $q$ is a neighbor of a subarc of $c \cap A_{i}$.

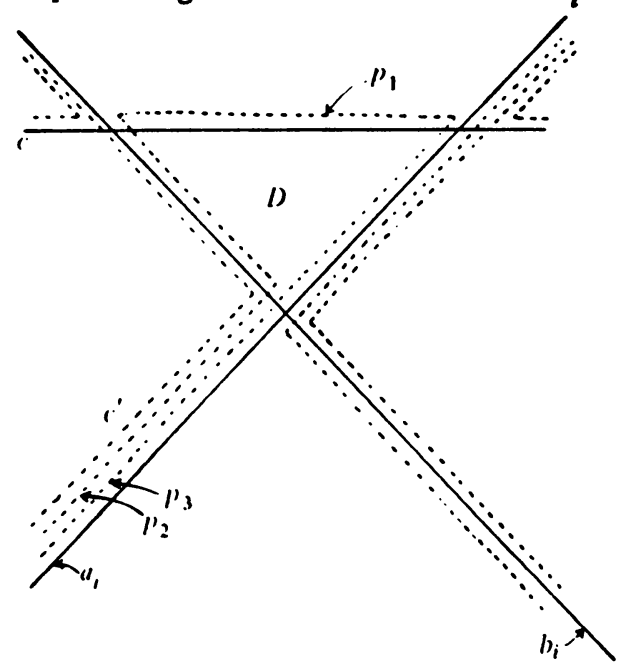

Figure 5

If we have previously performed Step 3 in this vicinity, there may in fact be a family of arcs in $c^{\prime}$ which are neighbors of $p$. Repeated application of the process just described moves each of these, beginning with the innermost one, 
to neighbors of $q$. After each construction we go back and check for possible applications of Steps 1 and 3.

The construction of the desired isotopy is completed when we have exhausted all the possibilities of applying the above four steps. Let $c$ "denote the final image of $c^{\prime}$ under this isotopy. Observe that if $c^{\prime} \cap c \neq \varnothing$ then $c^{\prime \prime} \cap c \neq \varnothing$. It is only during Step 2 that the complete removal of all points from $c^{\prime} \cap c$ could have been completed, and then only in the event that $c$ were to meet $a_{i}$ in a single point. However, this is not possible since $c$ is homologous to zero.

We are now in a position to look for a disk $E$ in $F$ such that $\partial E=s \cup t$, where $s$ is an arc in $c$ and $t$ is an $\operatorname{arc}$ in some $a_{i}$ or $b_{i}$ with $\partial t=\partial s=s \cap t$. We first eliminate the easy case. Suppose that in the construction of $c^{\prime \prime}$ we performed both Steps 2 and 4 across a pair of disks $D_{1}$ and $D_{2}$, respectively, which meet the same component of $A \cup B$. Since both Steps 2 and 4 were used, it follows that the disks $D_{1}$ and $D_{2}$ lie on the same side of either $a_{i}$ or $b_{i}$. Then the desired disk $E$ can clearly be found in $D_{1} \cup D_{2}$. Thus let us assume this situation does not occur.

Since $c^{\prime \prime}$ is isotopic to $c$ and $c^{\prime \prime} \cap c \neq \varnothing$, it follows from [6] that there exists a disk $D$ such that $D \cap\left(c \cup c^{\prime \prime}\right)=\partial D=p \cup q$, where $p$ and $q$ are arcs in $c$ and $c^{\prime \prime}$, respectively, with $p \cap q=\partial p=\partial q$. We see from the construction of $c^{\prime \prime}$ that $q \subset A \cup B \cup U$. We complete the proof by observing that there exists a disk $E$, corresponding to $D$, satisfying the required conditions.

Let us investigate the various ways in which the disk $D$ can arise. Keep in mind that there are two kinds of pieces of $c^{\prime \prime}$ which lie outside $A \cup B$. These are components of $d \cap \overline{(F-(A \cup B))}$ and arcs that are neighbors of components

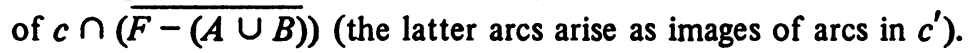

Case 1. The two points of $\partial q$ lie in a common component $V$ of $U \cap$ $(A \cup B)$ and $q$ meets only one component of $(\partial V) \cap(A \cup B)$. This situation is illustrated in Figure 6. Since $p \cup q$ bounds the disk $D$, the arc $q$ cannot consist totally of a union of neighboring pairs of arcs (as it does near $V$ ). Since $q \cap c=q \cap p=\partial q$, we see that $q$ must pass through the disk $A_{i} \cap B_{i}$, for some $i$, and meet both components of $\left(A_{i} \cup B_{i}\right)-\left(A_{i} \cap B_{i}\right)$. The only way in which the two pieces $t \cap\left(A_{i}-\left(A_{i} \cap B_{i}\right)\right)$ and $t \cap\left(B_{i}-\left(A_{i} \cap B_{i}\right)\right)$ can join up again is through a component of $c \cap \overline{(F-(A \cup B))}$ as shown in Figure 6. If we examine this situation carefully, we find that Step 4 can be applied here. Hence this case no longer occurs.

Case 2. The two points $p \cap q$ lie in the same component $V$ of $U \cap$ $(A \cup B)$ and $q$ meets two components of $(\partial V) \cap\left(A_{i} \cup B_{i}\right)$. Let us suppose that $V$ is a component of $U \cap\left(A_{i} \cup B_{i}\right)$. By the same reasoning as used in Case 1 , if $q$ meets both $A_{i}$ and $B_{i}$ then $q$ must either pass through $A_{i} \cap B_{i}$ or be the image of an arc in $c^{\prime}$ meeting $A_{i} \cap B_{i}$ under the isotopies of Steps 2 and 3. 


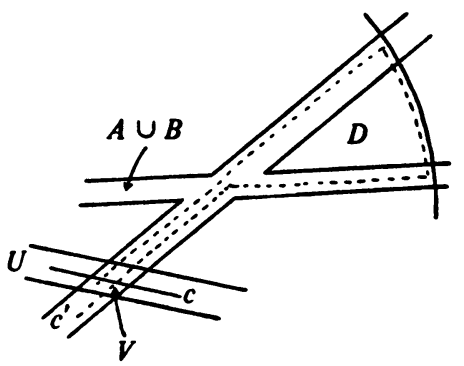

Figure 6

This situation is shown in Figure 7a. However, since $t\left(a_{i}\right)$ and $t\left(b_{i}\right)^{-1}$ have opposite sense, this situation cannot occur. Thus $q$ must meet either $A_{i}$ or $B_{i}$, but not both (see Figure 7b). The desired disk $E$ is easily found coinciding with $D$ on $F-(A \cup B)$.

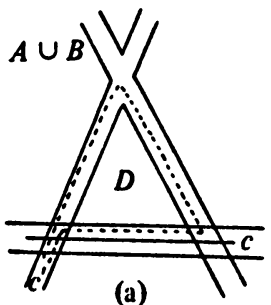

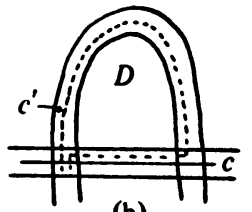

(b)

\section{Figure 7}

Case 3. The two points $\partial q$ lie in distinct components of $U \cap(A \cup B)$, say $V$ and $W$. If $V$ and $W$ are contained in the same component of either $A$ or $B$ (as shown in Figure 8a) then there clearly exists the sought after disk $E$, which again coincides with $D$ on $F-(A \cup B)$.

So let us suppose that $V$ and $W$ do not belong to the same component of $A$ or $B$. If $V \subset A_{i}$ and $W \subset B_{i}$ (for some $i$ ) then either $q \subset A_{i} \cup B_{i}$ or $q$ con-

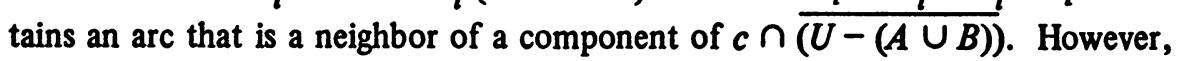
since this situation allows the application of either Step 2 or 3 , it does not occur. Thus $V$ and $W$ must be contained in distinct components of $A \cup B$. It follows that there is an arc in $c^{n}$ which is a neighbor of a component of $c \cap$ $(U-\overline{(A \cup B)})$ spanning the two components of $A \cup B$ (see Figure $8 \mathrm{~b}$ ). This also does not occur since Step 3 can be applied in such a situation.

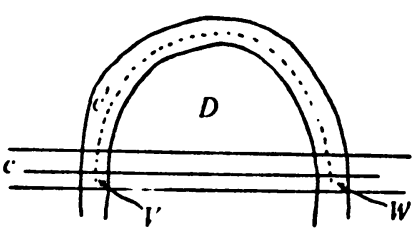

(a)

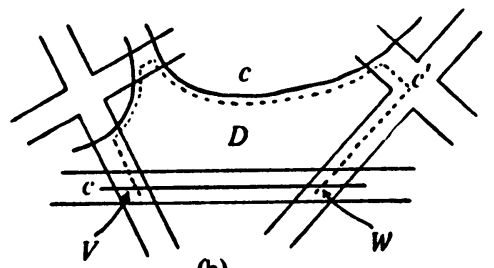

(b)

Figure 8 
We have considered all possible ways in which the disk $D$ can occur and have shown that either we have already eliminated the case or we can find the desired disk $E$. Notice that the disk $E$ found in Cases 2 and 3 meets only one of the curves $a_{i}$ and $b_{i}$. This completes the proof of the lemma.

LEMMA 11. The system of curves $\Gamma$ is isotopic in $F$ to a system $\Gamma_{1}$ such that $\alpha\left(\Gamma_{1}\right)$ is transverse to $\Gamma_{1}$ and there does not exist a disk $D$ such that $D \cap(x \cup \alpha(y))=\partial D=s \cup t$, where $\{x, y\} \subset\left\{a_{i}, b_{i}: 1 \leqslant i \leqslant g\right\}$, the arc $s$ is contained in $x$, the arc $t$ is contained in $\alpha(y)$, and $\partial t=\partial s=s \cap t$.

Proof. Move $\Gamma$ into $\alpha$-general position by an isotopy of $F$. Let us suppose that a.disk $D$, as described in the lemma, does exist. We may assume $D$ to be an innermost disk in the sense that no proper subset of $D$ is another such disk. We shall show that in the presence of this disk $D$ we can move $\Gamma$, by an isotopy, so as to decrease the number of points in $\alpha(\Gamma) \cap \Gamma$ and yet keep $\Gamma$ in $\alpha$-general position. There are three possible situations that might occur and each requires a slightly different treatment.

Let $U(s), U(t)$ denote small regular neighborhoods of $s, t$, respectively, such that $U(s) \cap U(t)$ is a regular neighborhood of $s \cap t$.

Case 1. Either $\alpha(s) \cap t=\varnothing$ or $\alpha(s)=t$. Choose an arc $t^{\prime}$ in $U(t)$ such that (i) $t^{\prime}$ is a neighbor of $t$, (ii) $t^{\prime} \cap(x \cup \alpha(y))=\partial t^{\prime} \subset x-s$, (iii) $t^{\prime}$ is transverse to $\Gamma-x$, and (iv) $\partial t \cup \partial t^{\prime}$ bounds two arcs $p$ and $q$ in $\overline{x-s}$. Define $\Gamma^{\prime}=$ $(\Gamma-(s \cup p \cup q)) \cup t^{\prime}$ and notice that $\Gamma^{\prime}$ is in $\alpha$-general position. Observe that $(\Gamma-x) \cap D$ is either empty or an arc, since $D$ is an innermost disk. Then $\Gamma^{\prime}$ is isotopic to $\Gamma$ and $\alpha\left(\Gamma^{\prime}\right) \cap \Gamma^{\prime} \subset \alpha(\Gamma) \cap \Gamma-(s \cap t)$.

Case 2. $\alpha(s) \cap t=\{d\}$, a single point in $\partial t$. Choose an arc $t^{\prime}$ in $U(t)$ such that (i) $t^{\prime}$ is a neighbor of $t$, (ii) $\partial t^{\prime}=d \cup e$, where $e \in x-s$, (iii) $(\partial t-d) \cup e$ bounds an arc $p$ in $\overline{x-s}$, (iv) $t^{\prime} \cap(x \cup \alpha(y))=\partial t^{\prime}$, and (v) $t^{\prime}$ is transverse to $\Gamma$ $-x$. Define $\Gamma^{\prime}=(\Gamma-(s \cup p)) \cup t^{\prime}$. Then $\Gamma^{\prime}$ is in $\alpha$-general position and is isotopic to $\Gamma$. We also have $\alpha\left(\Gamma^{\prime}\right) \cap \Gamma^{\prime} \subset \alpha(\Gamma) \cap \Gamma-(\partial t-d)$.

We can find the required system of curves $\Gamma_{1}$ by taking a system in $\alpha$ general position which is isotopic to $\Gamma$ and has the fewest number of points in $\alpha\left(\Gamma_{1}\right) \cap \Gamma_{1}$ among such systems.

LEMMA 12. Let $\Gamma=\Gamma_{1}$ satisfy the conclusion of Lemma 11. Suppose that the homeomorphism $\alpha \Phi \alpha^{-1}$ is homotopic to $\Phi$. Let $c_{1}$ be a simple closed curve separating $F$ such that $c_{1} \cap \alpha(\Gamma)=\varnothing$. Then there exists a simple closed curve $c$ homotopic to $c_{1}$ such that $c \cap(\Gamma \cup \alpha(\Gamma))=\varnothing$ and either $\alpha(c)=c$ or $\alpha(c) \cap c=\varnothing$.

Proof. We may assume that the curve $c_{1}$ meets $\Gamma$ transversally. Since $c_{1} \cap \alpha(\Gamma)=\varnothing$ we have $\alpha \Phi \alpha^{-1}\left(c_{1}\right)=c_{1}$. Thus $\Phi\left(c_{1}\right)$ is homotopic to $c_{1}$. It 
follows from Lemma 10 that there exists a disk $E$ such that $E \cap\left(c_{1} \cup x\right)=\partial E=$ $s \cup t$, where $x$ is some $a_{i}$ or $b_{i}, s$ is an arc in $c_{1}, t$ is an $\operatorname{arc}$ in $x$, and $s \cap t=\partial s$ $=\partial t$. Since $\Gamma$ satisfies the conclusion of Lemma 11, we see that $E \cap \alpha(\Gamma)=\varnothing$. Hence we can move $c_{1}$ across $E$ by an isotopy constant outside of a regular neighborhood of $E$ so as to decrease the number of points in $c_{1} \cap \Gamma$ by two. By repeating this construction we eventually obtain a simple closed curve $c_{2}$ isotopic to $c_{1}$ such that $c_{2} \cap(\Gamma \cup \alpha(\Gamma))=\varnothing$.

To complete the construction of $c$ we shall work with $c_{2} \cup \alpha\left(c_{2}\right)$ in F$(\Gamma \cup \alpha(\Gamma))$. First move $c_{2}$ into $\alpha$-general position. Among all simple closed curves in $F-(\Gamma \cup \alpha(\Gamma))$ which are homotopic to $c_{1}$ and in $\alpha$-general position, we may assume that $c_{2}$ has the fewest number of points in $c_{2} \cap \alpha\left(c_{2}\right)$.

If $c_{2} \cap \alpha\left(c_{2}\right)=\varnothing$ we set $c=c_{2}$. Otherwise, if $c_{2} \cap \alpha\left(c_{2}\right) \neq \varnothing$, we can find an innermost arc $s$ in $\alpha\left(c_{2}\right)$ with $s \cap c_{2}=\partial s$. Let $t_{1}$ and $t_{2}$ denote the two arcs $c_{2}-\partial s$. It follows from [6] that we may assume the simple closed curve $s \cup t_{1}$ bounds a disk in $F$. Thus $s \cup t_{2}$ is homotopic to $c_{2}$ (and disjoint from $\Gamma \cup \alpha(\Gamma))$. If $\alpha(s) \neq t_{2}$ then we proceed as we did in the proof of Lemma 11 to construct a new simple closed curve $c^{\prime}$ isotopic to $s \cup t_{2}$ with fewer points in $c^{\prime} \cap \alpha\left(c^{\prime}\right)$ than in $c_{2} \cap \alpha\left(c_{2}\right)$. Since this would contradict our choice of $c_{2}$, we must have $\alpha(s)=t_{2}$. We set $c=s \cup t_{2}$ and have $\alpha(c)=c$.

LEMMA 13. Let $\Gamma$ satisfy the conclusion of Lemma 11. Suppose the involution $\alpha$ induces the identity homomorphism on $H_{1}\left(F ; Z_{2}\right)$ and the homeomorphism $\alpha \Phi \alpha^{-1}$ is homotopic to $\Phi$. Then there exists a system $\left\{c_{1}, \ldots, c_{g-1}\right\}$ of pairwise disjoint and nonparallel, simple, closed curves such that $c_{i}$ separates $F, c_{i} \cap(\Gamma \cup \alpha(\Gamma))=\varnothing$, and $\alpha\left(c_{i}\right)=c_{i}($ for $1 \leqslant i \leqslant g-1)$.

Proof. Choose a set $\left\{c_{1}^{\prime}, \ldots, c_{g-1}^{\prime}\right\}$ of pairwise disjoint and nonparallel, simple, closed curves in the following manner. Each $c_{i}^{\prime}$ is to be chosen such that $c_{i}^{\prime}$ separates $F$ and $c_{i}^{\prime} \cap \alpha(\Gamma)=\varnothing$. First pick $c_{1}^{\prime}$ and apply Lemma 12 to obtain a simple closed curve $c_{1}$ homotopic to $c_{1}^{\prime}$ such that $c_{1} \cap(\Gamma \cup \alpha(\Gamma))=\varnothing$ and either $\alpha\left(c_{1}\right)=c_{1}$ or $\alpha\left(c_{1}\right) \cap c_{1}=\varnothing$. In the latter case there can be no handles between $\alpha\left(c_{1}\right)$ and $c_{1}$ since $\alpha$ induces the identity on $H_{1}\left(F ; Z_{2}\right)$. Thus there is an invariant annulus bounded by $\alpha\left(c_{1}\right) \cup c_{1}$ in which we can find an invariant closed curve parallel to $c_{1}$. Let us change our notation to denote this invariant curve by $c_{1}$ so that we have $\alpha\left(c_{1}\right)=c_{1}$. We repeat this process $g-1$ times, each time choosing $c_{i+1}^{\prime}$ to be disjoint from $c_{1} \cup \cdots \cup c_{i}$, to obtain the required system $\left\{c_{1}, \ldots, c_{g-1}\right\}$.

Proof of Theorem C. We suppose that $\alpha \Phi \alpha^{-1}$ is homotopic to $\Phi$ and show that this leads to a contradiction. It follows from Theorem $B$ that $\alpha$ induces the identity on $H_{1}\left(F ; Z_{2}\right)$. We note that $\alpha$ must be orientation preserving 
since, by Theorem B, the matrix for $\alpha_{*}: H_{1}(F ; Z) \rightarrow H_{1}(F ; Z)$ has the form of the matrix $A$ in Lemma 7. Hence the dimension of Fix $(\alpha)$ is strictly less than one. It follows from Lemma 13 that there exists a set $\left\{c_{1}, \ldots, c_{g-1}\right\}$ of simple closed curves such that $\alpha\left(c_{i}\right)=c_{i}$, the curve $c_{i}$ is disjoint from $\Gamma \cup \alpha(\Gamma)$, and (assuming we have chosen the notation correctly) the curve $c_{i}$ separates $a_{i} \cup b_{i}$ from $a_{i+1} \cup b_{i+1}$ (for $1 \leqslant i \leqslant g-1$ ).

Let $X$ denote the closure of the component of $F-\left(\bigcup_{i+1}^{g-1} c_{i}\right)$ which contains $a_{2} \cup b_{2}$. Then $X$ is homeomorphic to the torus $S^{1} \times S^{1}$ minus two open disks. Let $\hat{X}$ denote the torus obtained from $X$ by capping the two boundary curves with disks. We can find an arc $w$ properly embedded in $X-\alpha\left(a_{2} \cup b_{2}\right)$ that joins the two boundary components of $X$. Let $W$ denote a small regular neighborhood of $w \cup \partial X$ in $X$ such that $W$ is disjoint from $\alpha\left(a_{2} \cup b_{2}\right)$. Observe that the simple closed curve $c^{\prime}=\partial W$ is noncontractible in $X$ and separates $X$. Lemma 12 can be adapted to show there exists a simple closed curve $c$ homotopic to $c^{\prime}$ in $X$ such that $c \cap\left[\alpha\left(a_{2} \cup b_{2}\right) \cup\left(a_{2} \cup b_{2}\right)\right]=\varnothing$.

Since $\alpha\left(a_{2}\right)$ is homologous modulo 2 to $a_{2}$ in $F$ and hence in $X$, the curves $\alpha\left(a_{2}\right)$ and $a_{2}$ are homotopic in the torus $\hat{X}$. Recall [7, Proposition 5.1] that if two nonseparating, simple closed curves are homotopic in $S^{1} \times S^{1}$ then they are also homotopic in $S^{1} \times S^{1}-$ point . It follows that $\alpha\left(a_{2}\right)$ is homotopic to $a_{2}$ in $\hat{X}-c$ and hence in $X$. By a construction used in the proof of Lemma 12 we can find a simple closed curve $a_{2}^{\prime}$ homotopic to $a_{2}$ in $X$ such that $\alpha\left(a_{2}^{\prime}\right)=a_{2}^{\prime}$. Similarly, we can find a simple closed curve $b_{2}^{\prime}$ homotopic to $b_{2}$ such that $\alpha\left(b_{2}^{\prime}\right)=b_{2}^{\prime}$ is transverse to $a_{2}^{\prime}$. Since $a_{2}^{\prime}$ and $b_{2}^{\prime}$ meet in an odd number of points, there must be a fixed point of $\alpha$ contained in $a_{2}^{\prime} \cap b_{2}^{\prime}$. Here is our contradiction. Both of the curves $a_{2}^{\prime}$ and $b_{2}^{\prime}$ must contain a fixed point of $\alpha$ disjoint from $a_{2}^{\prime} \cap b_{2}^{\prime}$. But this requires that $\alpha$ have at least three fixed points in $X$, whereas there are only two.

Since the assumption that $\alpha \Phi \alpha^{-1} \simeq \Phi$ leads to this contradiction, we have proven that $\alpha \Phi \alpha^{-1} \neq \varnothing$ for any involution $\alpha$ of $F$.

\section{REFERENCES}

1. J. S. Birman and H. M. Hilden, On isotopies of homeomorphisms of Riemann sur. faces, Ann. of Math. (2) 97 (1973), 424-439. MR 48 \#305.

2. E. Bloomberg, Manifolds with no periodic maps, Trans. Amer. Math. Soc. 202 (1975), 67-78.

3. A. Borel, Seminar on transformation groups, Ann. of Math. Studies, no. 46, Princeton Univ. Press, Princeton, N. J., 1960, p. 38. MR 22 \#129.

4. P. E. Conner and F. Raymond, Manifolds with few periodic homeomorphisms, Proc. Second Conf. on Compact Transformation Groups (Amherst, Mass., 1971), part II, Springer-Verlag, New York, 1972, pp. 1-75.

5. P. E. Conner, F. Raymond and P. Weinberger, Manifolds with no periodic maps, Proc. Second Conf. on Compact Transformation Groups (Amherst, Mass., 1971), part II, Springer-Verlag, New York, 1972, pp. 81-108. 
6. D. B. A. Epstein, Curves on 2-manifolds and isotopies, Acta Math. 115 (1966), 83-107. MR 35 \#938.

7. K. W. Kwun and J. L. Tollefson, $P L$ involutions of $s^{1} \times s^{1} \times s^{1}$, Trans. Amer. Math. Soc. 203 (1975), 97-106.

8. W. B. R. Lickorisch, A representation of orientable combinatorial 3-manifolds, Ann. of Math. (2) 76 (1962), 531-540. MR 27 \#1929.

9. J. Nielsen, Abbildungsklassen endlicher Ordnung, Acta Math. 75 (1942), 23-115. MR 7, 137.

10. J. L. Tollefson, A 3-manifold with no PL involutions, Notices Amer. Math. Soc. 22 (1975), A-231. Abstract \#720-57-13.

DEPARTMENT OF MATHEMATICS, UNIVERSITY OF MICHIGAN, ANN ARBOR, MICHIGAN 48104

DEPARTMENT OF MATHEMATICS, UNIVERSITY OF CONNECTICUT, STORRS, CONNECTICUT 06268 